\title{
HRPD-X; a proposed upgrade to the ISIS High-Resolution Powder Diffractometer
}

\author{
AD Fortes and AS Gibbs
}

\section{Published version information}

Citation: AD Fortes and AS Gibbs. 'HRPD-X; a proposed upgrade to the ISIS HighResolution Powder Diffractometer.' Journal of Neutron Research, vol. 22, no. 2-3 (2020): 91-98. Is in proceedings of: 23rd Meeting of the International Collaboration on Advanced Neutron Sources (ICANS-XXIII), Chattanooga, Tennessee, United States, 13-18 Oct 2019.

DOI: $\underline{10.3233 / J N R-190130}$

The final publication is available at IOS Press through DOI above.

This version is made available in accordance with publisher policies. Please cite only the published version using the reference above. This is the citation assigned by the publisher at the time of issuing the AAM. Please check the publisher's website for any updates. 


\title{
1 HRPD-X; a proposed upgrade to the ISIS 2 High-Resolution Powder Diffractometer
}

\author{
3 A. D. Fortes ${ }^{\mathrm{a},{ }^{*}}$ and A. S. Gibbs ${ }^{\mathrm{a}^{*}}$ \\ $4 \quad{ }^{a}$ ISIS Pulsed Neutron and Muon Source, Rutherford Appleton Laboratory, Harwell Science and \\ 5 Innovation Campus, Chilton, Oxfordshire OX11 OQX, UK. \\ 6 E-mails: $\underline{\text { dominic.fortes@stfc.ac.uk, alexandra.gibbs@stfc.ac.uk }}$
}

Abstract. HRPD-X is a proposal to completely replace the current high-resolution powder diffractometer (HRPD) at the ISIS Neutron and Muon Source. The new instrument is expected to deliver a factor of four increase in solid-angle coverage. Taking advantage of new detector technology and coupled with a non-magnetic sample tank and improved incident- and diffractedbeam collimation, the new instrument will substantially improve HRPD's scientific capabilities to study magnetic structures and behaviour, high-pressure phenomena and supramolecular structures whilst strengthening its performance in alreadyestablished areas.

13 Keywords. High-resolution, neutron, powder diffraction, upgrade, ISIS

\section{Introduction}

The high-resolution powder diffractometer (HRPD) at the ISIS spallation neutron and muon source has been in operation for almost 35 years and remains one of the leading instruments of its kind in the world. With a $95 \mathrm{~m}$ primary flight path and backscattering detectors covering $2 \theta=158-176^{\circ}$ the instrument achieves an optimum $\Delta d / d \approx 3 \times 10^{-4}$ at the highest backscattering angles with $\Delta d / d \approx$ $6 \times 10^{-4}$ for data focussed over the whole backscattering array [1]. HRPD has an outstanding track record of high-impact science in the fields of phase transition studies, ab-initio structure determination, thermal expansion, microstructure analysis and a range of other fields in which subtle peaks shifts or splitting and peak-shape measurements are crucial [2]. The instrument has undergone numerous upgrades and refurbishments over the years, of which the most significant was the replacement of the original $m=1$ glass guide with an elliptical supermirror guide in 2007, realising a substantial flux increase principally at shorter wavelengths [3]. However, the complementary upgrades to the detector arrays required to capitalise on this upgrade of the guide did not occur.

In light of this and the continuing evolution of the scientific landscape, HRPD instrument scientists and ISIS design engineers developed a plan for a comprehensive upgrade to the instrument and its infrastructure. In spite of some delays and modifications to the design, the upgrade project - now dubbed "HRPD-X" - remains a bold proposal to significantly increase the scientific capabilities of the instrument and retain its world-leading position.

"Corresponding authors. E-mail: dominic.fortes@stfc.ac.uk / alexandra.gibbs@stfc.ac.uk 
The aim of the HRPD-X upgrade is to demolish the existing building in which HRPD is housed, erect a new building and then to install an entirely new instrument, replacing the old detector arrays, sample tank and incident-beam conditioning devices, but retaining the guide. The proposed detector arrays will be based on wavelength-shifting fibre technology, and cover a substantially larger solid angle than the current arrays, particularly in forward-scattering geometry which accesses longer $d$-spacings. Provision of a non-magnetic sample tank will allow HRPD-X to carry out measurements in applied magnetic fields up to $10 \mathrm{~T}$. Furthermore, improvements in upstream beam conditioning and sample collimation will work to reduce the comparatively large divergence of the supermirror guide and reduce backgrounds from in-situ sample environment devices.

This short paper will outline the current configuration of HRPD, summarise the scientific drivers for the upgrade specifications and provide some details of the proposed HRPD-X instrument.

\section{Current instrument configuration}

HRPD occupies port S8 on ISIS target station 1 (TS-1), viewing a centrally-poisoned cryogenic $\mathrm{CH}_{4}$ moderator. Neutrons propagate along an initially straight path through the shutter and target shielding (all lined with $m=3$ supermirror-coated glass), and are then transported out to $L=93.5 \mathrm{~m}$ along a curved elliptical guide, described in more detail by Ibberson [3]. Directly after the guide exit, a pair of horizontal and vertical $\mathrm{B}_{4} \mathrm{C}$ jaws are used to condition the incident beam before it passes through a beam monitor and ultimately encounters the sample position at $L=95 \mathrm{~m}$.

The geometry of the present detector arrangement is illustrated in Figure 1 and detailed in Table 1. The backscattering detectors comprise a circular array, split into eight wedge-shaped octants. The neutrons are detected by $5 \mathrm{~mm}$-wide strips of $\mathrm{ZnS}: \mathrm{Ag} /{ }^{6} \mathrm{LiF}$-scintillator coupled by clear plastic fibres to singlechannel photomultiplier tubes (PMTs) [4]. These modules were installed in 1994, replacing the instrument's original $1984 \mathrm{Li}$-glass detector modules in which the detector-element pitch was $15 \mathrm{~mm}$. Since 2007, the top and bottom octants have been excluded from the reduced data used for analysis due to the larger vertical divergence introduced by the new guide. Following a replacement of the backscattering PMTs in 2019 (see below), the exposed fibre ends in these two vertical octants (numbers 1 and 5) were covered over with $\mathrm{Al}$ plates, rendering the omission of these data a permanent feature.

Two banks of detectors are installed at $2 \theta=90 \pm 10^{\circ}$, one on the north side of the sample tank and one on the south side. These were fitted in 1999, replacing a single array of detectors on the north side that had been in operation since 1988. Like the backscattering modules, these consist of $\mathrm{ZnS}: \mathrm{Ag} /{ }^{6} \mathrm{LiF}-$ scintillator modules with a $3 \mathrm{~mm}$ pitch, coupled by clear fibres to single-channel PMTs [4].

In forward scattering there is a small array of $1 / 2$-inch helium tubes covering $2 \theta$ from $28-32^{\circ}$. These tubes were recycled from a previous installation on another instrument and installed in 1992. They are mounted in a $3 \times 3$ array of modules, each module containing eight tubes mounted vertically with a centre-to-centre separation between the tubes of $24 \mathrm{~mm}$.

The main sample tank consists of a simple cuboid box, with a volume of $\sim 0.39 \mathrm{~m}^{3}$, comprised of $1 "$ thick stainless steel. A circular aperture, or Tompkinson flange, on the top of the tank allows mounting of all standard sample-environment equipment. Upstream, there is a conical extension of the vacuum tank up to the backscattering detectors. Orthogonal to the incident beam are large evacuated wing tanks 
that carry the diffracted beam under vacuum to the 90-degree detectors. A downstream aperture leads into a slightly larger secondary tank $\left(0.43 \mathrm{~m}^{3}\right)$; early in the instrument's history this provided a second sample position $2 \mathrm{~m}$ from the backscattering detectors, which partially offset the coarse $15-\mathrm{mm}$ elements in the original Li-glass detector modules by measuring samples at a higher effective $2 \theta$. However, this secondary position has not been used for over 20 years. On the north side of the downstream tank is a vacuum window that allows forward-scattered neutrons to propagate through an aluminium box filled with argon gas and then into the low-angle detector bank.

Fig. 1. Diffracted-beam geometry of the current HRPD instrument; lines are drawn in the illuminated areas in increments of $2^{\circ}$ in $2 \theta$.

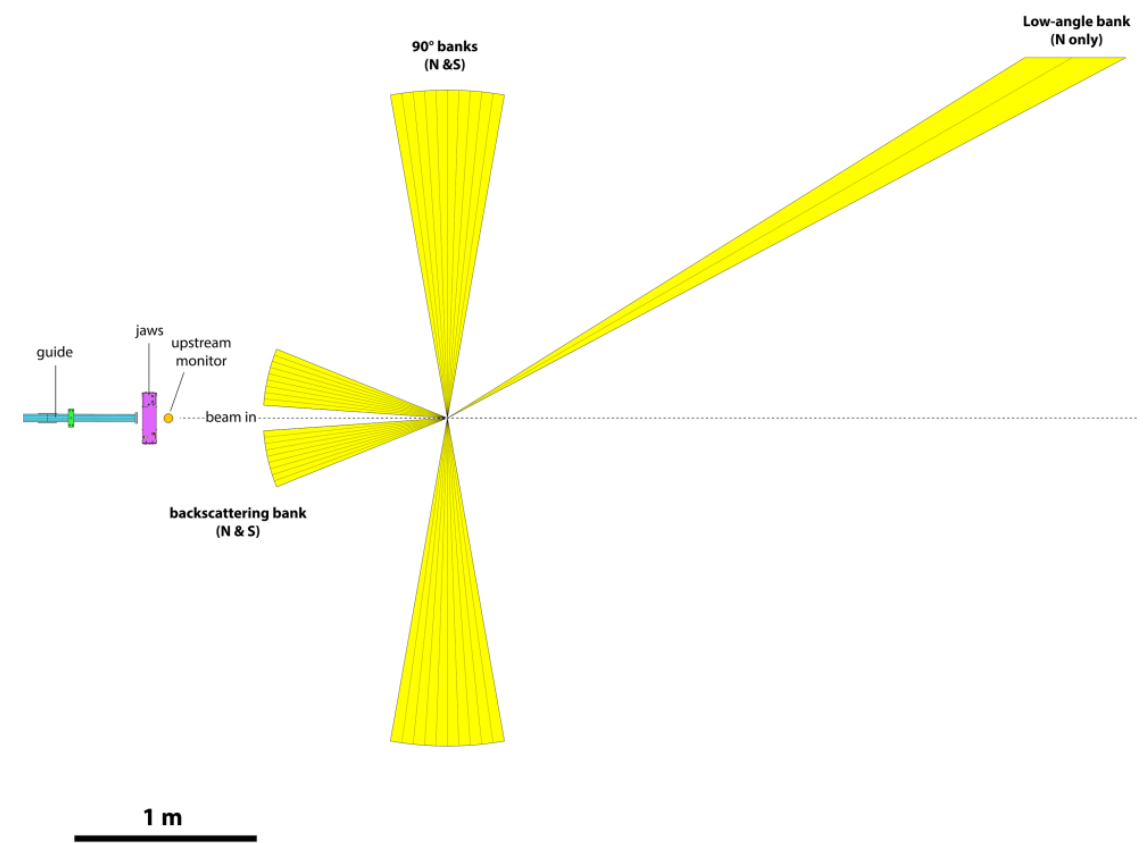

10

11 Table 1. Data on the coverage and resolution of HRPD's current detector modules.

\begin{tabular}{l|ccc}
\hline & $\begin{array}{c}\text { Bank 1 } \\
\text { Backscattering }\end{array}$ & $\begin{array}{c}\text { Bank 2 } \\
\mathbf{9 0 ~ d e g r e e s}\end{array}$ & $\begin{array}{c}\text { Bank 3 } \\
\text { Low-angle }\end{array}$ \\
\hline$\Delta d / d$ & $\sim 6 \times 10^{-4} \dagger$ & $\sim 2 \times 10^{-3}$ & $\sim 1 \times 10^{-2}$ \\
$2 \theta$ & $167.3 \pm 8.8^{\circ}$ & $90.0 \pm 9.6^{\circ}$ & $29.9 \pm 2.3^{\circ}$ \\
$\varphi$ & $\pm 67.5^{\circ}$ & $\pm 25.7^{\circ}$ & $\pm 13.5^{\circ}$ \\
$L 2 \min (\mathrm{m})$ & 1.010 & 1.805 & 3.710 \\
$L 2 \max (\mathrm{m})$ & 1.010 & 1.805 & 4.285 \\
Total area $\left(\mathrm{m}^{2}\right)$ & 0.32 & 1.81 & 0.38 \\
Solid angle $(\mathrm{sr})$ & 0.318 & 0.598 & 0.0095 \\
\hline
\end{tabular}

$\dagger$ Full-bank rather than optimal resolutions are quoted. 
It should be noted that the original generation of detectors in backscattering and at 90 -degrees were replaced after 10 years of operation but that those replacements are now 25 and 20 years old, respectively. Moreover, the recycled low-angle bank is now 27 years old. Coupled with old power supply and data-acquisition electronics, this has led to a substantial decrease in reliability and increase in electronic noise over recent years, with a significant risk that the crucial backscattering array would experience major failures before an upgrade of the instrument could be implemented in the early 2020s. As a result, 96 new PMTs were installed on octants 2-4 and 6-7 of the backscattering array, and the electronics for the backscattering and $90^{\circ}$ banks were replaced over the summer of 2019 , with those for low-angle bank to be completed in Spring 2020. The stipulation that all new electronic equipment must be located in an air-conditioned 'counting house' necessitated a concomitant refurbishment of the user area, with a temporary cabin being mounted externally to the west wall of HRPD's brick building; a new doorway and extended mezzanine connect the cabin to the existing instrument platform.

\section{The case for an upgrade}

In order to maintain a globally competitive position at the forefront of high-resolution neutron powder diffraction, HRPD must build on its early 1990s track record of adopting new detector technology and adapting to the changing needs of the scientific community.

There is a general trend towards studying more complex materials, typically with larger unit cells, which includes supramolecular frameworks (MOFs, zeolites, clathrates), materials that develop superstructures, modulated structures and complex magnetic structures. In HRPD's range of available wavelength bands, the relevant structural information (e.g., superlattice peaks, magnetic reflections) are best observed at moderate to low $2 \theta$ values so our first upgrade specification is to increase the instrument's coverage at $2 \theta<90^{\circ}$, whilst retaining the current performance at high $2 \theta$. This has the additional benefit of eliminating gaps in $d$-spacing coverage that currently appear in longer time-offlight windows.

There is another trend in the direction of carrying out more complex 'complementary' or 'in-situ' measurements, which might colloquially be described as "powder neutron diffraction plus something else done simultaneously" where the 'something else' could be electrical transport measurements, resonant ultrasound spectroscopy, Raman spectroscopy, heat capacity measurements, and so forth. Since this often introduces additional material in the vicinity of the sample, be it support structures or measurement devices, there is thus a need for improved collimation of both the incident beam (to limit over-illumination) and of the diffracted beam. Likewise, a developing need to make high-pressure measurements at the highest possible resolution places even more strict demands on the collimation by virtue of the small sample size compared with the size of the pressure-generating devices. Our second specification is thus to minimise overall instrumental backgrounds and cut as much parasitic scattering as possible from complex sample-environment equipment by introducing a radial collimator around the sample position. This specification is also highly relevant for other measurements of small sample volumes, due to e.g. high-pressure synthesis or other synthetic techniques in which homogeneity between batches cannot necessarily be guaranteed.

HRPD's current sample tank is too small simply to insert a radial collimator of the kind used on GEM and Polaris, so this objective in turn mandates the provision of a new larger vacuum tank. Since it is also the case that the scientific community wishes increasingly to make high-resolution neutron powder 
diffraction measurements under an applied magnetic field, which the current stainless-steel vacuum tank precludes, our third specification is that the new vacuum tank must be fabricated from nonmagnetic materials and the PMTs be appropriately shielded.

\section{The HRPD-X upgrade plan}

Following a successful upgrade to the ISIS high-intensity diffractometer Polaris in 2012 [5], a plan was developed to adopt a similar tank and detector architecture for an upgrade to HRPD. This involved a large increase in the solid angle coverage, with two new large detector banks replacing the small lowangle bank, a Polaris-style ( $c a .20 \mathrm{~m}^{3}$ ) vacuum tank and a Polaris-style radial collimator drum. Technical designs and McStas-based optimizations were carried out, which formed the basis for the 2014/15 HRPD Instrument Development Plan and International Review. Since that time, small modifications have been made, principally to the secondary flight paths of the proposed detector array to find improvements in resolution (most notably in bank 3), and further McStas simulations have been performed primarily to assess the effect on beam divergence of adding additional upstream collimation.

The most recent iteration of the proposed upgrade design involves an array of wavelength-shifting fibre detector elements [6] positioned on a logarithmic spiral in $2 \theta$ such that $L_{2}=A \cdot \exp (-B \cdot 2 \theta)$, where $A=$ $4000 \mathrm{~mm}$ and $B=0.43 \mathrm{rad}^{-1}$; these are to be coupled to 64-channel PMTs. The precise geometry of the proposed array is shown in Figure 2 and summarised in Table 2. The upgraded detector complement covers a solid angle four times larger than the current instrument $(1.185 \pi$ sr versus $0.295 \pi \mathrm{sr})$ and almost $2 / 3$ the coverage of Polaris $(1.805 \pi \mathrm{sr})$. The proposed geometry - specifically the longer secondary flightpaths - coupled with a finer pitch of detector elements in backscattering, leads to expected improvements in resolution over the current instrument, as shown in Figure 3. This large active area $\left(17.5 \mathrm{~m}^{2}\right)$ poses design challenges in terms of running the fibres along Debye-Scherrer arcs whilst also having the detector surface curved, or at worst a polygonal approximation thereof, in both $2 \theta$ and $\varphi$.

Nevertheless, the upgraded detectors will ensure better count-rate matching between banks and eliminate gaps in $d$-spacing that occur in the current detector array when using longer time-of-flight windows (Figure 4).

The original intention to have a $20 \mathrm{~m}^{3}$ vacuum tank, with most of the secondary flight paths in vacuum resulted in two significant problems. The principal difficulty was the dimensions of the vacuum windows in front of the detector modules, which would have needed to be extremely large and require a great deal of invasive support structure. Secondly, the large tank would have comparatively long pumpdown times, even with a dry-air let-up system, and it would be inconveniently time-consuming to achieve the high vacuum needed to run closed-cycle refrigerators without introducing a separate cryogenic vacuum bin, adding to the background and the diffracted beam attenuation. Our solution to this, at present, is to design a non-magnetic sample tank that is only slightly larger than the current HRPD vacuum tanks but which can nonetheless host a Polaris-style radial collimator, and to propagate the diffracted beam between the vacuum windows and the detectors through tanks filled with natural argon. This is essentially the same arrangement HRPD uses today on its forward-scattering bank, where the diffracted neutrons pass through $\sim 1.26 \mathrm{~m}$ of vacuum, $2.0-2.7 \mathrm{~m}$ of argon and $0.3-0.4 \mathrm{~m}$ of air (with a $1.2 \mathrm{~mm}$ vacuum membrane and two $50 \mu \mathrm{m}$-foil gas-tank windows), and is similar to the design employed on WISH. In this scenario, the vacuum windows have unsupported areas of $0.2-0.4$ 
$1 \mathrm{~m}^{2}$, which compares favourably with the smaller windows currently in use on HRPD $\left(0.08-0.27 \mathrm{~m}^{2}\right)$

2 and is much smaller than the secondary vacuum windows on the current $90^{\circ}$ detectors $\left(1.04 \mathrm{~m}^{2}\right)$.

3 Fig. 2. Provisional diffracted-beam geometry of the proposed HRPD-X instrument; lines are drawn in 4 the illuminated areas in increments of $2^{\circ}$ in $2 \theta$.

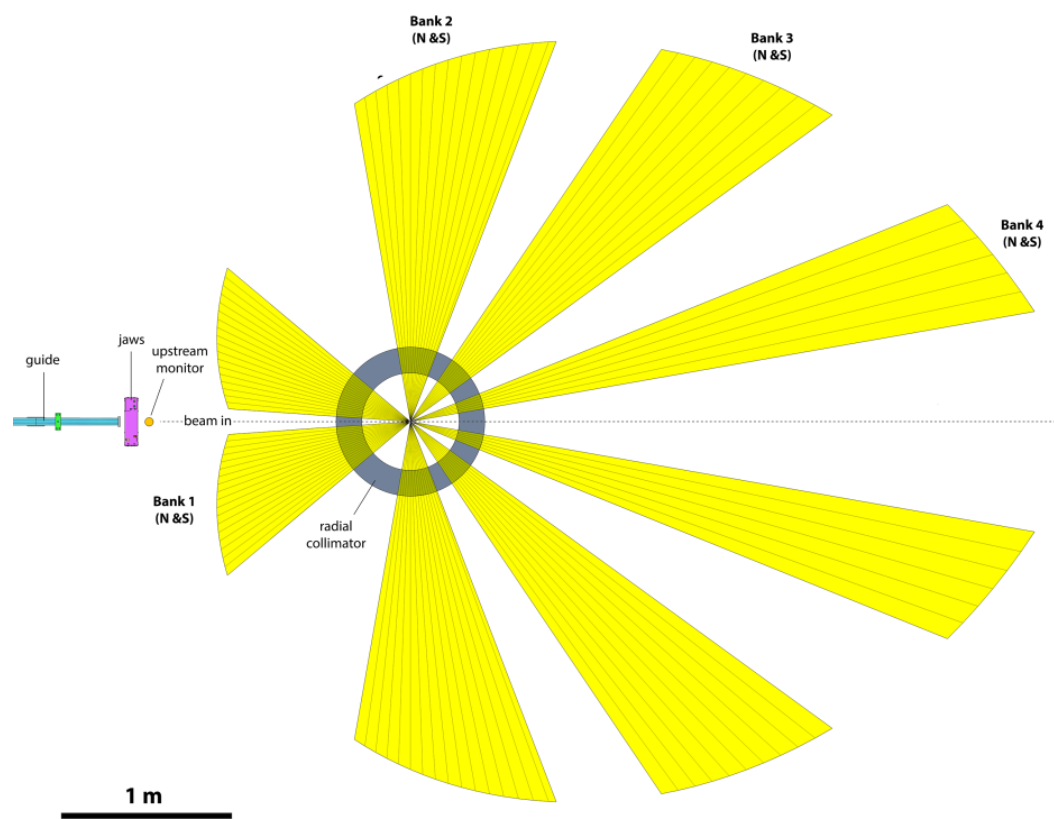

7 Table 1. Data on the coverage and resolution of the HRPD-X detector modules.

\begin{tabular}{l|cccc}
\hline & $\begin{array}{c}\text { Bank 1 } \\
\text { Backscattering }\end{array}$ & $\begin{array}{c}\text { Bank 2 } \\
\text { 90 degrees }\end{array}$ & $\begin{array}{c}\text { Bank 3 } \\
\text { Intermediate }\end{array}$ & $\begin{array}{c}\text { Bank 4 } \\
\text { Low-angle }\end{array}$ \\
\hline Est. $\Delta d / d$ & $\sim 6 \times 10^{-4} \dagger$ & $\sim 2 \times 10^{-3}$ & $\sim 3.5 \times 10^{-3}$ & $\sim 1.5 \times 10^{-2}$ \\
$2 \theta$ & $158 \pm 18^{\circ}$ & $84.5 \pm 15.5^{\circ}$ & $46 \pm 10^{\circ}$ & $16 \pm 6^{\circ}$ \\
$\varphi$ & $\pm 85^{\circ}$ & $\pm 40^{\circ}$ & $\pm 33^{1 / 3^{\circ}}$ & $\pm 70^{\circ}$ \\
$L 2 \min (\mathrm{m})$ & 1.068 & 1.889 & 2.628 & 3.391 \\
$L 2 \max (\mathrm{m})$ & 1.399 & 2.383 & 3.053 & 3.711 \\
Total area $\left(\mathrm{m}^{2}\right)$ & 2.217 & 6.877 & 4.728 & 3.674 \\
Solid angle (sr) & 1.374 & 1.486 & 0.581 & 0.282 \\
\hline
\end{tabular}

$\dagger$ Full-bank rather than optimal resolutions are quoted. 
1 Fig. 3. Calculated $\Delta d / d$ resolution for HRPD-X banks $1-3$ compared with the current instrument's backscattering (bank 1) and 90-degree (bank 2) detectors; assumed sample diameter $=6 \mathrm{~mm}$.

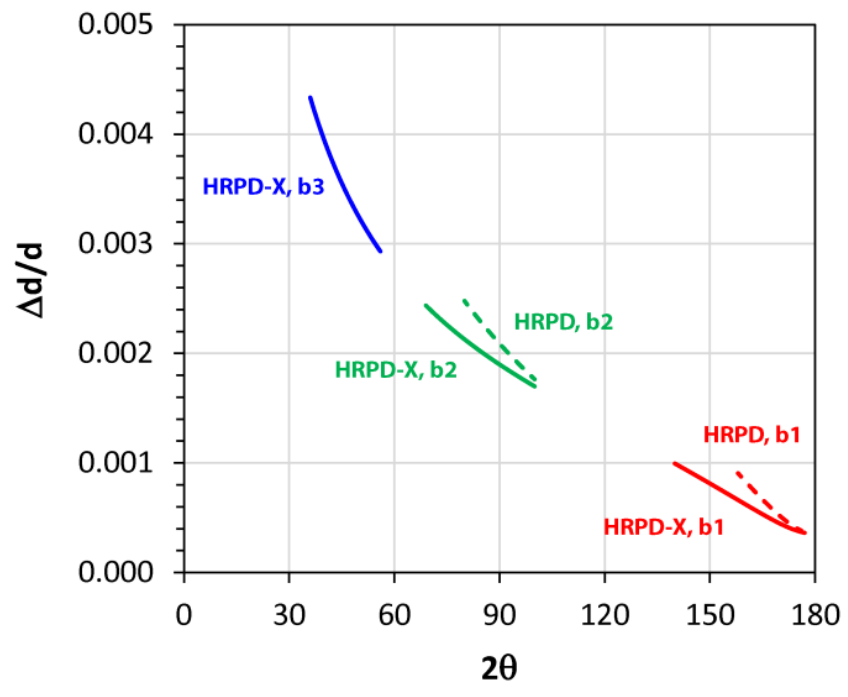

5 Fig. 4. Available $d$-spacing ranges for the various 'standard' time-of-flight windows operated on 6 HRPD; (a) the current instrument and (b) for HRPD-X. Note the gap in coverage that appears between 7 banks 2 and 3 for the 100-200 ms window in the current instrument configuration.
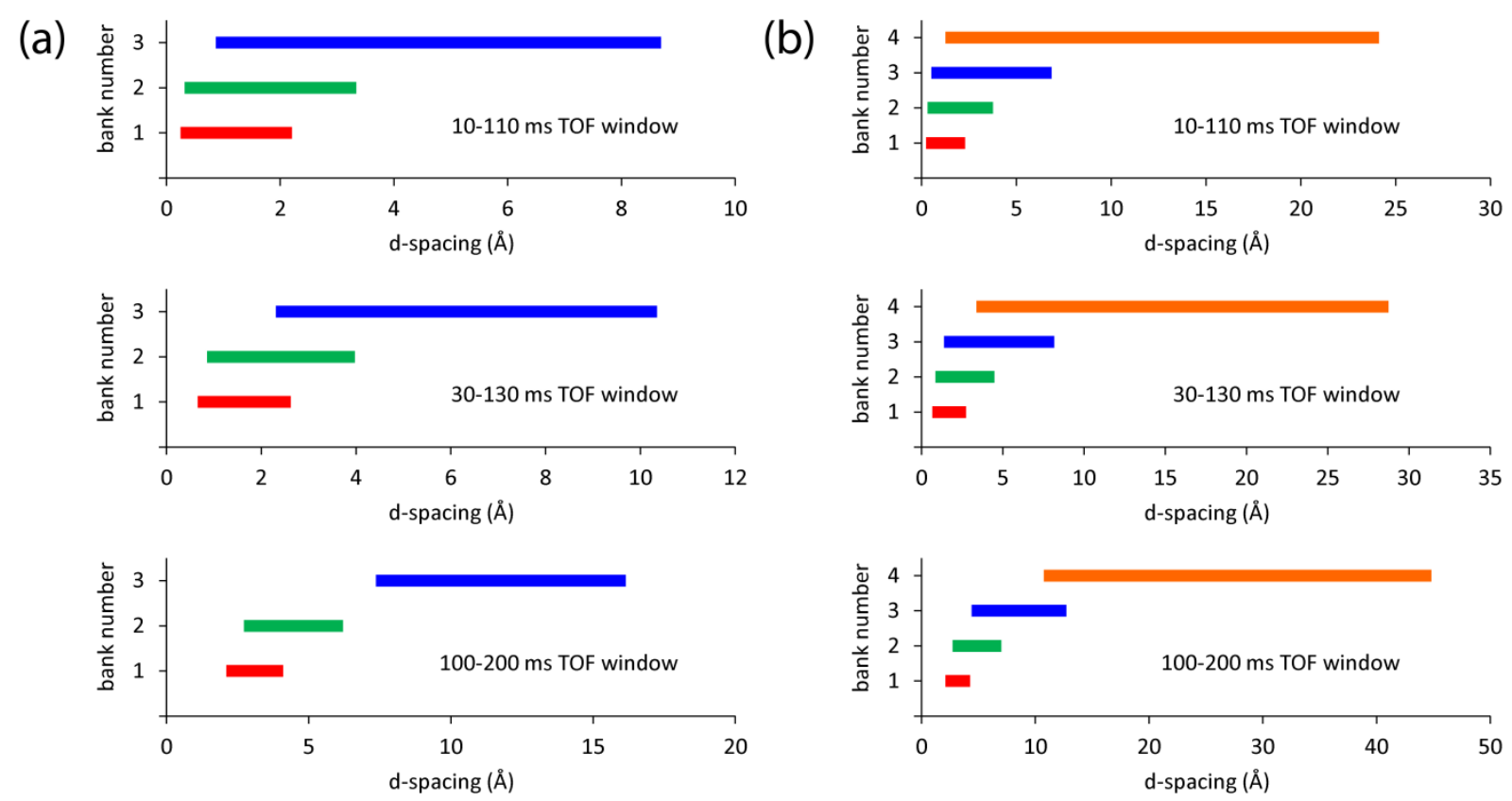
Whilst striving to minimise alterations to the existing supermirror guide, work has been carried out to evaluate what may be done to reduce the divergence of the incident beam whilst simultaneously preserving beam homogeneity, flux and resolution. HRPD currently has two pairs of $\mathrm{B}_{4} \mathrm{C}$ jaws centred $\sim 70 \mathrm{~mm}$ downstream of the guide exit window, which are typically used to define a rectangular aperture $20 \mathrm{~mm}$ high and $15 \mathrm{~mm}$ wide; the final evacuated section of the flight-line then contains a fixed rectangular $\mathrm{B}_{4} \mathrm{C}$ collimation snout with a $40 \times 20 \mathrm{~mm}$ aperture located $450 \mathrm{~mm}$ in front of the sample position. Various configurations of additional rectangular apertures were tested in McStas simulations; the results indicate that a second set of moveable jaws at $L=92.3 \mathrm{~m}$ (i.e., $1 \mathrm{~m}$ upstream of the guide exit) achieves our goals of maximising beam homogeneity, reducing divergence by $45 \%$ and keeping $60 \%$ of the flux at $\lambda=2 \AA$.

Since the new instrument cannot be accommodated inside the present brick building (designated R69), the initial upgrade plan included the provision of a new steel-framed building of considerably larger dimensions. The location of HRPD (Figure 5) in a 'notch' between the second target-station building (R80), an array of electrical workshops (R6a), and the TS-2 extracted-proton beam (R6-R80 link), with a roadway passing around the eastern end R69 that must be navigable by fire engines and other large articulated vehicles, places significant constraints on how much the footprint of the building may be enlarged, and indeed on the instrument geometry itself: the instrument must fit within a square box that extends no more than $5.5 \mathrm{~m}$ downstream of the guide exit and $2.75 \mathrm{~m}$ either side of the beam centreline.

Fig. 5. Geographical constraints on the HRPD upgrade instrument and building arise from the proximity of nearby building and the need for large articulated vehicles to move around the area (large dashed arrows), with a minimum lateral clearance of $5 \mathrm{~m}$. HRPD and its guide tunnel are shaded black, other buildings in dark grey, and raised off-road areas in light grey.

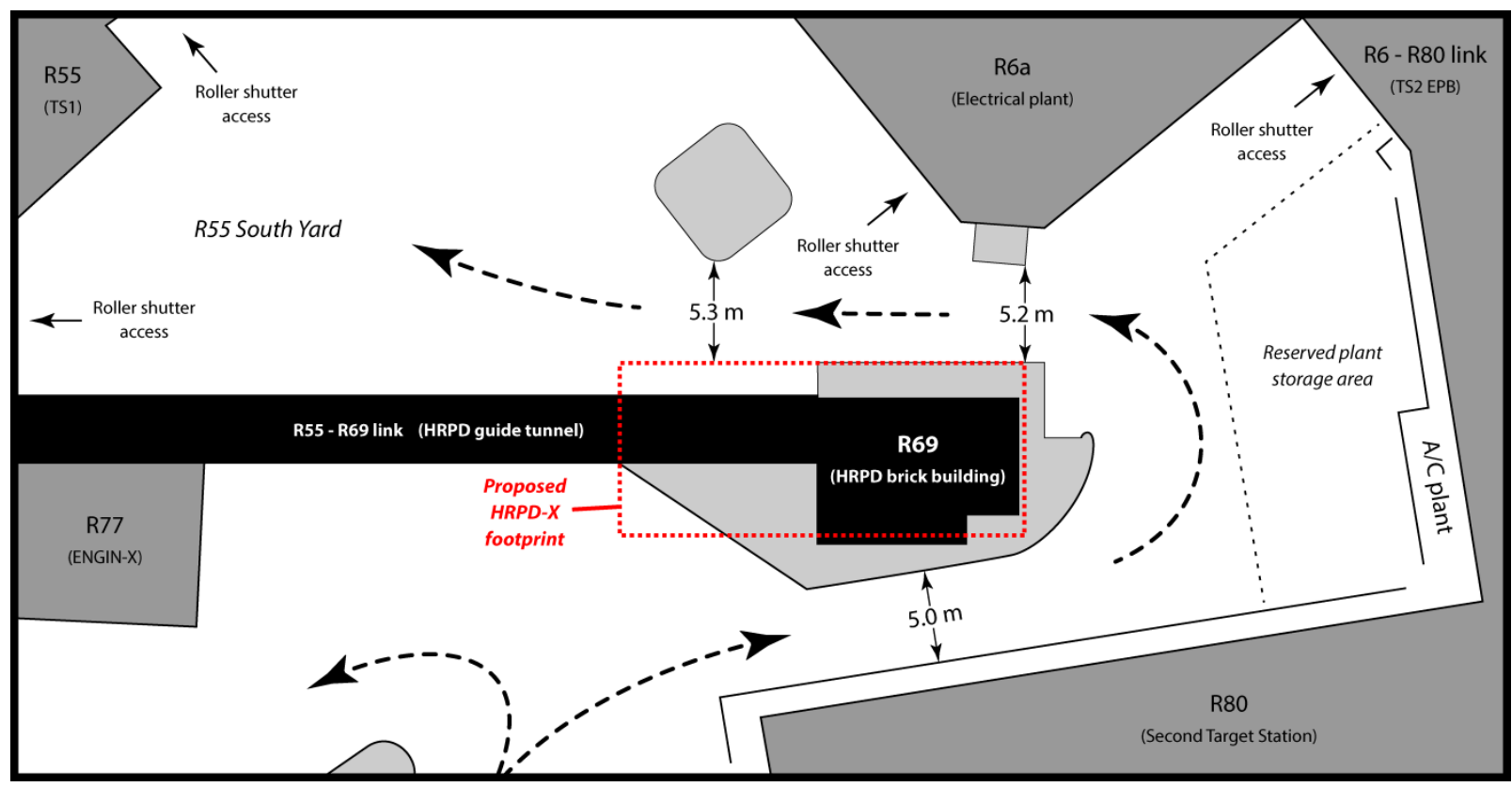


The initial 2014 upgrade building design, with a large shielded blockhouse around the new instrument, created a significant pinch-point on the SE corner, reducing the width of the road between the building and the crash-barrier adjacent to TS-2 down to $3.1 \mathrm{~m}$. We have since reviewed the way in which the area around the instrument itself should be shielded and interlocked, whilst aiming to maintain safety and accessibility for detector maintenance, and have been able to design a building with a substantially smaller footprint that avoids the vehicular pinch-points. This $196.5 \mathrm{~m}^{2}$ two-story building is designed to be fully wheelchair accessible to cater for users with diverse mobility needs, and includes $52 \mathrm{~m}^{2}$ of space for instrument control and data analysis, $87 \mathrm{~m}^{2}$ of mezzanine floor around the instrument sample pit, to include sample-preparation laboratory space, and approximately $35 \mathrm{~m}^{2}$ to house all of the instrument's electronics.

\section{Timescales and summary}

The detector arrays, the vacuum/gas tanks, and the building have recently moved into the detailed design phase, anticipating the release of funds in the time-period after ISIS Target-Station 1 undergoes its refurbishment (Sept. 2020 - Dec. 2021) [7]. To prevent further disruption to the HRPD user programme it is anticipated that the instrument will continue to operate in its current configuration for at least 1 year after the TS-1 project. The current instrument would be dismantled and the building demolished around the beginning of 2023, with the expectation that construction of the new building should be complete in early 2024 and the new instrument installed and fully commissioned before HRPD's $40^{\text {th }}$ birthday in December 2024. The anticipated overall cost of the upgrade will be $\sim £ 9 \mathrm{M}$.

The proposed developments outlined in this paper will allow HRPD's exceptional capabilities to be applied to a range of new scientific problems, including supramolecular frameworks, complex magnetic structures and multi-GPa high-pressure studies whilst adding considerably to the extent and quality of data obtained in the instrument's day-to-day range of, e.g., parametric variable-temperature studies, variable-composition studies, and precise crystal structure refinements.

Acknowledgements We would like to acknowledge the significant volume of work undertaken on the HRPD upgrade project by previous instrument scientists and researchers (including K. S. Knight, W.I.F. David, R. M. Ibberson, A. Daoud-Aladine and J. Jacas Biendicho) along with critical input from other members of the Crystallography Group (in particular S. Hull) and engineering and technical staff at ISIS (in particular D. McPhail, S. Waller, N. Rhodes and G. J. Sykora).

\section{References}

[1] R. M. Ibberson et al., The High Resolution Powder Diffractometer (HRPD) at ISIS: A User guide. RAL Technical Report 92-031 (1992).

[2] https://www.isis.stfc.ac.uk/Pages/Hrpd-publications.aspx

[3] R. M. Ibberson, Nucl. Instr. Methods A, 600, 47-49 (2009)

[4] N. Rhodes et al., Nucl. Instr. Methods A, 529, 243-248 (2004)

[5] R. Smith et al., Rev. Sci. Instr. 90, 115101 (2019)

[6] G. J. Sykora et al., ICANS XXIII proceedings, abstract 114 (2019)

[7] S. Gallimore and D. Haynes, ICANS XXIII proceedings, abstract 12 (2019) 